\title{
Generalized Plasmonic Modelling of the Effect of Refractive Index on Laser-Induced Periodic Nanostructures
}

\author{
A. Abdelmalek ${ }^{1}$, Z. Bedrane ${ }^{1}$, V. Bharadwaj ${ }^{2}$, E.-H. Amara ${ }^{3}$, R. Ramponi ${ }^{2}$, S. M. Eaton ${ }^{2}$ \\ 1. Physics Department, Theoretical Physics Laboratory, Tlemcen University, Tlemcen 13000, Algeria \\ 2. IFN-CNR and Dipartimento di Fisica, Politecnico di Milano, 20133 Milano, Italy \\ 3. Centre de Développement des Technologies Avancées, CDTA, PO. Box 17 Baba-Hassen, Algiers 16303, \\ Algeria \\ E-mail: ahmed.abdelmalek@univ-tlemcen.dz
}

Received: 24 August 2019; Accepted: 1 September 2019; Available online: 5 November 2019

\begin{abstract}
Laser-induced periodic surface structures (LIPSS) have been studied theoretically employing generalized plasmonic modelling on several dielectric materials such as $\mathrm{SiO}_{2}, \mathrm{Al}_{2} \mathrm{O}_{3}, \mathrm{ZnO}$, AlAs and diamond exposed to $800 \mathrm{~nm}$ wavelength multi-pulse femtosecond laser irradiation. The study of the optical properties of the materials during laser irradiation reveals a formation of a metallic like pseudo-material on the irradiated layer during excitation. A study of the grating periodicity of the nanostructures shows that the materials having a high refraction index allow LIPSS formation with a wide range of grating periodicities. Results also show High Spatial Frequency LIPSS formation with periodicities 3 to 8 times lower than the laser wavelength.
\end{abstract}

Keywords: Nanostructure; LIPSS; Refractive index; Plasmonic model.

\section{Introduction}

Laser-induced periodic surface structures (LIPSS), also called as nanogratings [1] or nanoripples [2] has become a fascinating phenomenon in laser-matter interaction science. Ultrashort laser pulses on the order of femtosecond duration can alter the morphology of material surface through the LIPSS formation [3]. Depending on the irradiation conditions, two types of LIPSS can be observed. In the first type, Low Spatial Frequency LIPSS (LSFL) with period, $\Lambda$ between $\lambda / 2$ and $\lambda$, where $\lambda$ is the wavelength of the incident laser. This type of LIPSS generally occurs at metal surface or semiconductor with a laser fluence near the ablation threshold, where the interference of the electric field of the Surface Plasmon Polariton (SPP) with the incident laser beam leads to a deposition of spatially modulated energy on the material resulting in a nanostructure formation [4]. In the second type of LIPSS, a sub-wavelength periodic nanostructure can be observed at dielectric surface during the irradiation by multi-pulse femtosecond laser below the ablation threshold. This phenomenon is called High Spatial Frequency LIPSS (HSFL), having a period $\Lambda$ less than $\lambda / 2$ [5]. This kind of LIPSS has been shown to improve the optical performance of materials like diamond [6].

Bonse et al. [4] observed two kinds of LIPSS on titanium (Ti) surface after multi-pulse femtosecond laser irradiation with $\lambda=790 \mathrm{~nm}$ wavelength; LSFL with period $\Lambda$ between 510 and $670 \mathrm{~nm}$, which agree with the interference model and HSFL with periods close to $\lambda / 10$. Pan et al. [7] studied LIPSS formation on the surface of niobium (Nb). It was reported that as the number of applied pulses increases, the LSFL nanostructure transforms to the HSFL type. Since the HSFL type formation is based on disorder phenomenon, which is a fundamental process for nanoablation [5], and because the disordering occurs only in materials with an energy band-gap [8], this type of nanostructure cannot be observed in metals. This HSFL type appears only in dielectrics and semiconductor surfaces during excitation by multipulse fs-laser irradiation [3]. We believe the appearance of HSFL nanostructure on metals such as titanium may be due to the oxidation of the surface during the irradiation, where the superficial layer of titanium or niobium gets oxidized to dielectric forms such as $\mathrm{TiO}$ [9] or NbO [10].

El-Bandrawy et al. [11] studied experimentally the formation of LIPSS on silicon surface during ultrashort laser pulse irradiation with $800 \mathrm{~nm}$ wavelength. The results clarified that the interference model is only valid for nanostructure formation of LSFL type which has periods $\Lambda>\lambda / 2$. Therefore, the HSFL nanostructure cannot be explained by an interference phenomenon because its periods are much less than $\lambda / 2$. Miyaji et al. [12] showed that a metal-like layer can be formed at the surface of dielectric materials during the irradiation by multi-pulse fslaser due to disordering (non-thermal melting) [8]. Therefore, they have shown that the excitation of SPP is the dominant process for the HSFL type nanograting formation with fs-laser pulses [13]. 
In our previous work [5], we proposed that the origin of the HSFL nanostructure could be due to the combination of non-thermal fusion and plasmonic excitation during multi-pulse fs-laser irradiation. It has been shown that the period of nanogratings depends on the incident laser wavelength [5]. An increasingly wider range of nanostructure periodicity was theoretically predicted as the irradiation wavelength was increased from $200 \mathrm{~nm}$ to $900 \mathrm{~nm}$ in study of femtosecond laser exposure of gallium nitride $(\mathrm{GaN})$ [14]. In the present work, we study the significant effect of the refractive index on the range of periodicity of the nanostructures. We calculate the HSFL-type nanostructure periodicity observed in various materials based on our generalized plasmonic model [5]. With our model, we can accurately predict the periodicity range of the nanograting from the direct fs-laser writing at any dielectric material surface. We have demonstrated that the nanostructure periodicity range increases with the material refractive index.

\section{Theoretical model}

Since a pseudo-metal layer is built on the surface of dielectric materials during the irradiation by multipulse fslaser, a generalized plasmonic model can be used to model the formation of nanostructure between pseudo-metal layers and dielectric surrounding medium in various dielectric materials such as $\mathrm{SiO}_{2}, \mathrm{Al}_{2} \mathrm{O}_{3}$, $\mathrm{ZnO}$, AlAs and diamond under $800 \mathrm{~nm}$ multi-pulse fs-laser irradiation. Air is considered as the dielectric environment.

The surface plasmon polariton (SPP) dispersion relation is given by [15]:

$$
\omega_{s p}^{2}=c^{2} k_{s p}^{2} \frac{\left(\varepsilon_{p m}+\varepsilon_{d}\right)}{\varepsilon_{p m} \varepsilon_{d}}
$$

where $\omega_{s p}$ is the SPP frequency, $c$ is the speed of light in vacuum, $\varepsilon_{p m}$ is the dielectric function of pseudo-metal layer and $k_{s p}=k_{s p 1}+i k_{s p 2}$ is the plasmon propagation number with the real part $\mathcal{R}\left(k_{s p}\right)=k_{s p 1}=2 \pi / \lambda_{s p}=$ $\pi / \Lambda$ and the imaginary part $\operatorname{im}\left(k_{s p}\right)=k_{s p 2}$, where $\lambda_{s p}$ is the plasmon wavelength and $\Lambda$ is the period of nanostructures and $\varepsilon_{d}$ is the dielectric constant of the surrounding material medium. This dielectric constant is approximated as $\varepsilon_{d}=m \varepsilon_{\text {bulk }}+(1-m) \varepsilon_{\text {air }}$, where $m$ represent the factor of mixed states, depending implicitly on the number of incident laser pulses, which takes the value 0 (air/ pseudo-metal) or 1 ( pseudo-metal/bulk), $\varepsilon_{\text {air }}=1$ and $\varepsilon_{\text {bulk }}=n^{2}$ is obtained from literature for the various materials at $800 \mathrm{~nm}$ wavelength (see Table 1 )

[16]. The dielectric function of the pseudo-metal layer is given by the Drude model [17]:

$$
\varepsilon_{p m}=\varepsilon_{p m 1}+i \varepsilon_{p m 2}=1+\left(\varepsilon_{\mathrm{bulk}}-1\right)\left(1-\frac{n_{e h}}{n_{0}}\right)-\frac{\omega_{p}^{2}}{\omega^{2}} \frac{1}{1+\frac{i}{\omega \tau_{e e}}}
$$

With

$$
\begin{aligned}
& \varepsilon_{p m 1}=1+\left(\varepsilon_{\mathrm{bulk}}-1\right)\left(1-\frac{n_{e h}}{n_{0}}\right)-\frac{\omega_{p}^{2}}{\omega^{2}} \frac{1}{1+\frac{1}{\omega^{2} \tau_{e e^{2}}}} \\
& \varepsilon_{p m 2}=\frac{1}{\omega \tau_{e e}} \frac{\omega_{p}^{2}}{\omega^{2}} \frac{1}{1+\frac{1}{\omega^{2} \tau_{e e}{ }^{2}}}
\end{aligned}
$$

where $n_{0}$ is the electron concentration in the valence band (we consider a value of $10^{23} \mathrm{~cm}^{-3}$ ), $\tau_{e e}$ the electronelectron collision time ( $1 \mathrm{fs}), n_{e h}$ is electron-hole plasma density, $\omega$ is laser frequency and

$$
\omega_{p}=\sqrt{\frac{n_{e h} e^{2}}{\varepsilon_{0} m_{o p t}^{*} m_{e}}}
$$

is the plasma frequency, where $m_{\text {opt }}^{*}$ is optical effective mass [18] (Table 1).

Table 1. Data of optical properties of various materials used in the simulations at $800 \mathrm{~nm}$ wavelength, obtained from literature $[16,19]$.

\begin{tabular}{llllll}
\hline & $\mathrm{SiO}_{2}$ & $\mathrm{Al}_{2} \mathrm{O}_{3}$ & $\mathrm{ZnO}$ & Diamond & $\mathrm{AlAs}$ \\
\hline$n$ & 1.4533 & 1.7601 & 1.9591 & 2.4048 & 3.0044 \\
$m_{\text {opt }}^{*}$ & $0.18[18]$ & $0.3[20]$ & $0.318[21]$ & $0.3[5]$ & $0.5[22]$ \\
$R_{i}$ & 0.034143 & 0.075844 & 0.10505 & 0.17023 & 0.25 \\
\hline
\end{tabular}

From Eq.1 and Eq. 2, we can obtain the following generalized plasmonic model: 


$$
\begin{aligned}
& K_{s p 1}=\frac{1}{\sqrt{2}} \frac{\omega}{c}\left[\frac{\gamma \alpha}{\alpha^{2}+\beta^{2}}+\frac{\gamma}{\left(\alpha^{2}+\beta^{2}\right)^{1 / 2}}\right]^{1 / 2} \\
& K_{s p 2}=\frac{\omega^{2}}{2 c^{2} K_{s p 1}} \frac{\gamma \beta}{\alpha^{2}+\beta^{2}}
\end{aligned}
$$

where $\alpha=\varepsilon_{p m 1}{ }^{2}+\varepsilon_{p m 2}{ }^{2}+\varepsilon_{d} \varepsilon_{p m 1}, \beta=\varepsilon_{d} \varepsilon_{p m 2}$ and $\gamma=\varepsilon_{d}\left(\varepsilon_{p m 1}{ }^{2}+\varepsilon_{p m 2}{ }^{2}\right)$.

For more details see Ref. [5]. This model was simulated using MATLAB software.

\section{Results and discussion}

\subsection{Optical properties during fs-laser excitation}

The fs-laser irradiation of a dielectric material increases the density of free electrons in the conduction band which causes the dielectric constant to become a dielectric function dependent on the electronic excitation density.

Figure 1 shows the dielectric function of the materials irradiated by multipulse fs-laser. The dielectric function decreases with increased irradiation (number of pulses). It can be seen that the dielectric function becomes negative when the electron density $n_{e h}$ exceeds the critical density of the plasmonic excitation $n_{c r}$ at which $\mathcal{R} \varepsilon_{p m}\left(n_{c r}\right)=$ 0 . In this regime, it can be inferred that the femtosecond laser has the capability to form a layer with metallic characteristics during the irradiation. The critical density of plasmonic excitation is given in Table 2.

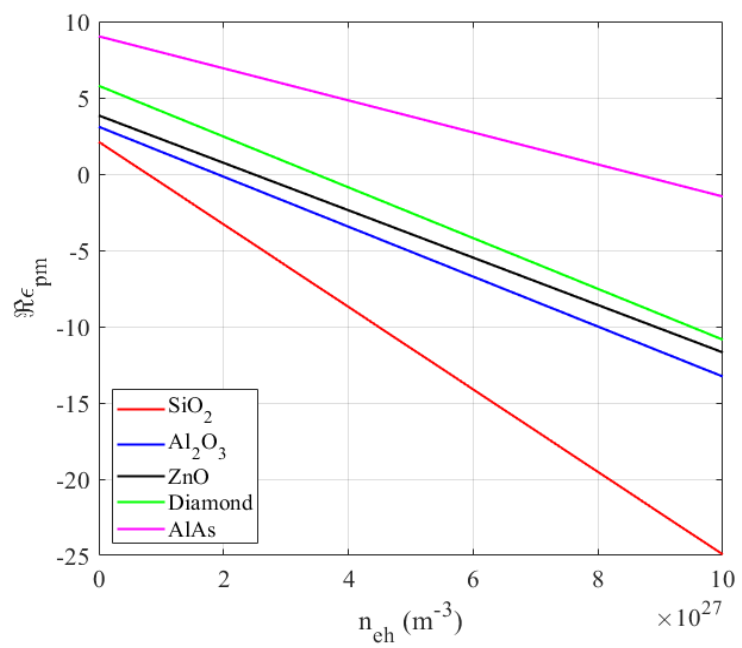

Fig. 1. Dielectric function of the pseudo-metal layer as a function of excited electron-hole plasma density.

Table 2. The critical density of plasmonic excitation at $800 \mathrm{~nm}$ wavelength.

\begin{tabular}{llllll}
\hline Materials & $\mathrm{SiO}_{2}$ & $\mathrm{Al}_{2} \mathrm{O}_{3}$ & $\mathrm{ZnO}$ & Diamond & $\mathrm{AlAs}$ \\
\hline$n_{c r} \times 10^{27}$ & 0.8 & 1.9 & 2.5 & 3.4 & 8.6 \\
\hline
\end{tabular}

The decrease of dielectric function affects the optical properties of the irradiated material surface, such as the reflectivity, as shown in Figure 2 with

$$
R=\frac{(n-1)^{2}+\kappa^{2}}{(n+1)^{2}+\kappa^{2}}
$$

where,

$$
n=\frac{1}{\sqrt{2}}\left[\varepsilon_{p m 1}+\left(\varepsilon_{p m 1}^{2}+\varepsilon_{p m 2}^{2}\right)^{1 / 2}\right]^{1 / 2}
$$

is the real part of the refractive index and $\kappa=\varepsilon_{p m 2} / 2 n$ is the absorption coefficient.

It can be seen from Figure 2 that the reflectivity increases dramatically with increase in irradiation because the excited electron plasma creates a metal-like material and hence, the surface plasmon can be excited during the irradiation. Note that the reflectivity of material surface with no irradiation shown in Figure 2 matches well with the value from literature given in Table 1, validating the effectiveness of this model. 


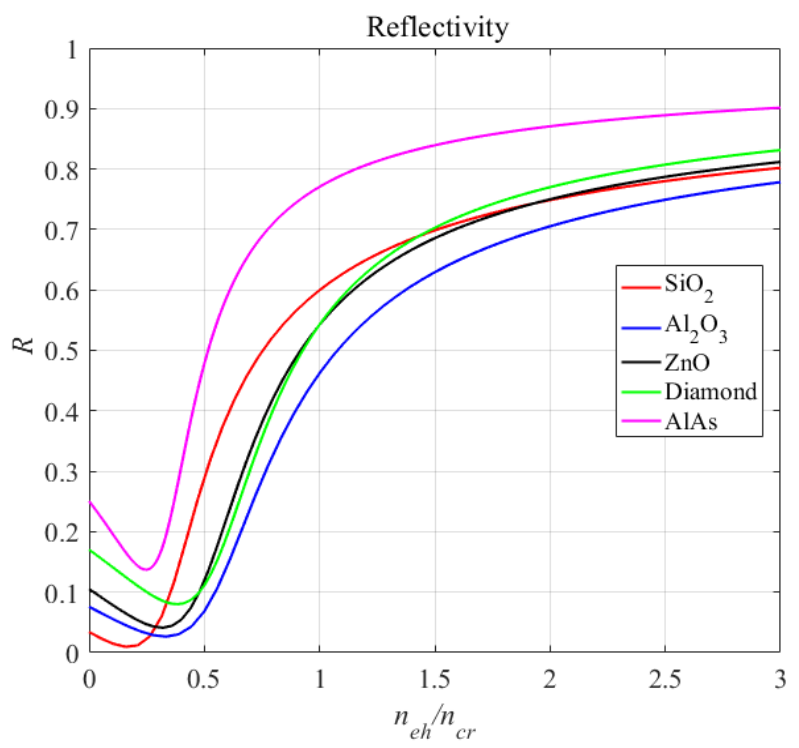

Fig. 2. Reflectivity of the pseudo-metal layers during the irradiation as a function of excited electron density at $800 \mathrm{~nm}$ wavelength.

\subsection{Determination of HSFL grating periods}

A polished single crystal optical grade CVD diamond [23] has been irradiated by Light Conversion Pharos amplified femtosecond laser delivering $230 \mathrm{fs}$ pulses at $1030 \mathrm{~nm}$ wavelength with a repetition rate of $250 \mathrm{kHz}$. The laser was incident normal to the target surface and focused with a 0.42 NA (50×) microscope objective, with a spot size of approximately $1.6 \mu \mathrm{m}$. The laser polarization was perpendicular to the scan direction and the experiment was carried out in air. Figure 3 shows scanning electron microscopy (SEM) image taken on the irradiated diamond surface for the selected wavelength under 114 pulses ( $3.5 \mathrm{~mm} / \mathrm{s} \mathrm{scan}$ speed) and $17 \mathrm{~mW}$ of power. We observe a regular nanostructure induced by direct fs-laser writing with period $215 \pm 5 \mathrm{~nm}$, about 5 times lower than laser wavelength.

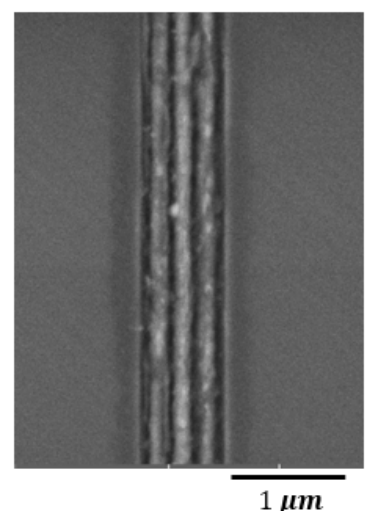

Fig. 3. SEM image of diamond surfaces irradiated with femtosecond laser pulses perpendicularly polarized to the scan direction for $v=3.5 \mathrm{~mm} / \mathrm{s}$ and $\mathrm{P}=17 \mathrm{~mW}$ at $1030 \mathrm{~nm}$ wavelength.

Based on the generalized plasmonic model presented above, we can determine the range of grating periodicities of HSFL nanostructures that can be observed during multi-pulse femtosecond laser irradiation on various dielectric materials having different refractive indices. Figure 4 shows the evolution of LIPSS period for various materials during the excitation of electron-hole plasma at two interface; air/pseudo-metal, where the minimal surface plasmonic resonance $\mathrm{RP}_{\text {min }}$ can be excited since $\varepsilon_{p m}=-\varepsilon_{\text {air }}(m=0)$; and pseudo-metal/bulk, where the maximal surface plasmonic resonance $\mathrm{RP}_{\max }$ to be excited since $\varepsilon_{p m}=-\varepsilon_{b u l k}(m=1)$. At minimal plasmonic resonance, the First Ordered Nanostructure (FON) with period $\Lambda_{\text {FON }}$ can be constructed at the surface. When the excited electron density increases, the period of nanostructure decreases and the Last Ordered Nanostructure (LON) with period $\Lambda_{\text {LON }}$ appear, owing to intense evanescent wave due to increase in plasmonic frequency.

The data shown in the Table 3 are derived from the Figure 4 where FON and LON correspond to $\mathrm{RP}_{\min }$ and $\mathrm{RP}_{\max }$ respectively. 


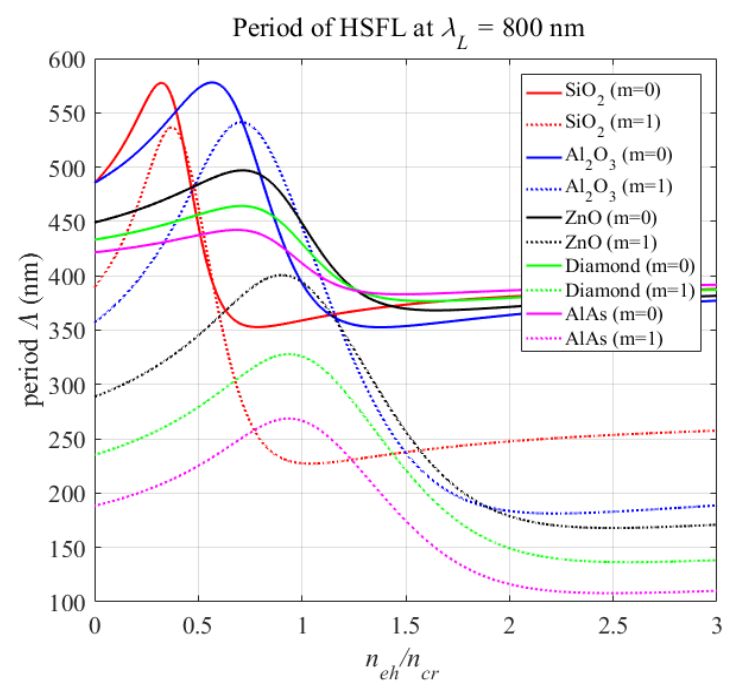

Fig. 4. The evolution of LIPSS period for different materials during the excitation of electron density at two interfaces air/irradiated layer $(m=0)$ and irradiated layer/bulk material $(m=1)$.

Table 3. The values of FON and LON periods derived from Figure 4 . $\Delta \Lambda$ represent the range of nanograting for each materials.

\begin{tabular}{llll}
\hline Materials & $\Lambda_{\mathrm{FON}}(\mathrm{nm})$ & $\Lambda_{\mathrm{LON}}(\mathrm{nm})$ & $\Delta \Lambda=\Lambda_{\mathrm{FON}}-\Lambda_{\mathrm{LON}}$ \\
\hline $\mathrm{SiO}_{2}$ & 378.8 & 239.9 & 138.9 \\
$\mathrm{Al}_{2} \mathrm{O}_{3}$ & 352.8 & 181.9 & 170.9 \\
$\mathrm{ZnO}$ & 396.6 & 180.2 & 216.4 \\
$\mathrm{Diamond}$ & 389.2 & 149.3 & 239.9 \\
$\mathrm{AlAs}$ & 396.8 & 116.3 & 280.5 \\
\hline
\end{tabular}

Based on the data in Table 3, we can obtain Figure 5, which shows the range of grating periodicities $\Lambda$, of HSFL type nanostructures that can be observed by direct femtosecond laser writing in different materials. We can notice that the range of grating periodicities increases when the refractive index increases. At the same laser wavelength, aluminum arsenide (AlAs) shows a large window of $280.5 \mathrm{~nm}$ which allows the experimentalist to observe nanostructures with periods between 116.3 and $396.8 \mathrm{~nm}$, whereas in silica, there is a narrow window of $138.9 \mathrm{~nm}$. It can also be observed that the first ordered nanostructure (FON) is slightly less than $\lambda / 2$ and almost constant for all materials. On the other side, the period of ( $\mathrm{LON})$ is approximately $\lambda / 3$ for low refractive index materials like silica $\left(\mathrm{SiO}_{2}\right)$ awhile is on the order of $\lambda / 8$ for high refractive index materials like aluminum arsenide (AlAs).

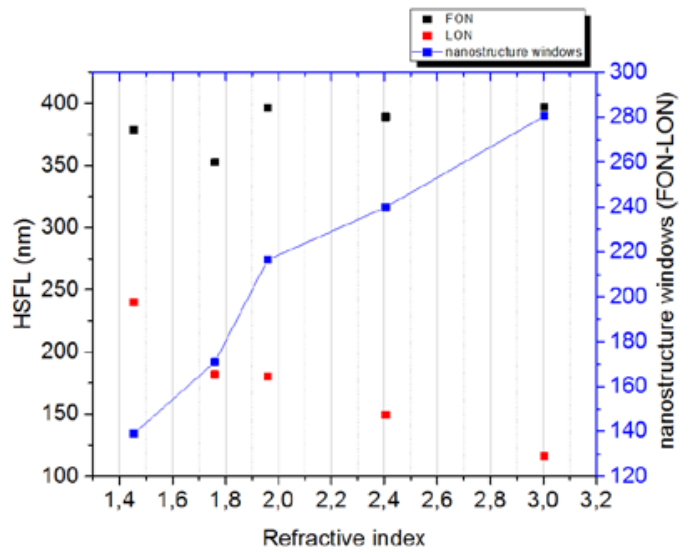

Fig. 5. HSFL periods and nanostructure windows as a function of refractive index.

\section{Conclusions}

The generalized plasmonic modelling has been used to study the formation HSFL-type nanostructures on the surface of dielectric materials irradiated by femtosecond laser at $800 \mathrm{~nm}$ wavelength. The study performed on the 
optical properties such as the dielectric function and the reflectivity of the dielectric materials reveal the metallic like characteristics on the surface, because of the intense electronic excitation during the irradiation. The range of HSFL nanostructure grating periodicity on various dielectric materials was also determined and it can be concluded that this range is broad for materials having a high refractive index. The modelling will also allow the study of nano-grating formation in black diamond with applications in solar cell micro devices.

\section{Acknowledgements}

The researchers are grateful for support from the ERC project PAIDEIA GA n.816313, MIUR SIR project Diamante and H2020 Marie Curie ITN project PHOTOTRAIN. IFN thanks Prof. Guglielmo Lanzani and Dr. Luigino Criante for the use of the FemtoFab facility at CNST-IIT Milano. Shane Eaton of IFN is grateful for support through the CNR Short Term Mobility (STM) program.

\section{References}

[1] Hnatovsky C, Taylor RS, Rajeev PP, Simova E, Bhardwaj VR, Rayner DM, Corkum PB. Pulse duration dependence of femtosecond-laser-fabricated nanogratings in fused silica. Applied Physics Letters. 2005;87(1):014104.

[2] Le Harzic R, Dörr D, Sauer D, Neumeier M, Epple M, Zimmermann H, Stracke F. Formation of periodic nanoripples on silicon and germanium induced by femtosecond laser pulses. Physics Procedia. 2011;12:2936.

[3] Bonse J, Höhm S, Kirner SV, Rosenfeld A, Krüger J. Laser-induced periodic surface structures—A scientific evergreen. IEEE Journal of Selected Topics in Quantum Electronics. 2016;23(3):9000615.

[4] Bonse J, Krüger J, Höhm S, Rosenfeld A. Femtosecond laser-induced periodic surface structures. J. Laser Appl. 2012;24(4):042006.

[5] Abdelmalek A, Sotillo B, Bedrane Z, Bharadwaj V, Pietralunga S, Ramponi R, Amara EH, Eaton SM. Origin of femtosecond laser induced periodic nanostructure on diamond. AIP Advances. 2017;7(10):105105.

[6] Calvani P, Bellucci A, Girolami M, Orlando S, Valentini V, Lettino A, Trucchi DM. Optical properties of femtosecond laser-treated diamond. Applied Physics A. 2014;117(1):25-29.

[7] Pan A, Dias A, Gomez-Aranzadi M, Olaizola SM, Rodriguez A. Formation of laser-induced periodic surface structures on niobium by femtosecond laser irradiation. Journal of Applied Physics. 2014;115(17):173101.

[8] Zier T, Zijlstra ES, Kalitsov A, Theodonis I, Garcia ME. Signatures of nonthermal melting. Structural Dynamics. 2015;2(5):054101.

[9] Kofstad P. High-temperature oxidation of titanium. Journal of the Less Common Metals. 1967;12(6):449464.

[10] Pons M, Caillet M, Galerie A. High temperature oxidation of niobium superficially coated by laser treatment. Materials Chemistry and Physics. 1987;16(5-6):423-432.

[11] El-Bandrawy M, Bugayev AA. Nanoscale self-assembled surface structures of silicon at femtosecond excitation. Journal of Laser Applications. 2007;19(3):155-157.

[12] Miyaji G, Kobayashi W, Miyazaki K. Reflectivity change in nanoscale modification of DLC film with femtosecond laser pulses. J. Laser Micro Nanoeng. 2007;2:146-151.

[13] Miyaji G, Miyazaki K. Origin of periodicity in nanostructuring on thin film surfaces ablated with femtosecond laser pulses. Optics Express. 2008;16(20):16265-16271.

[14] Miyaji G, Miyazaki K. Fabrication of 50-nm period gratings on GaN in air through plasmonic near-field ablation induced by ultraviolet femtosecond laser pulses. Optics Express. 2016;24(5):4648-4653.

[15] Kawata S, Ohtsu M, Irie M, editors. Near-field optics and surface plasmon polaritons. Springer Science \& Business Media; 2001.

[16] www.refractiveindex.info

[17] Sokolowski-Tinten K, von der Linde D. Generation of dense electron-hole plasmas in silicon. Physical Review B. 2000;61(4):2643.

[18] Vexler MI, Tyaginov SE, Shulekin AF. Determination of the hole effective mass in thin silicon dioxide film by means of an analysis of characteristics of a MOS tunnel emitter transistor. Journal of Physics: Condensed Matter. 2005;17(50):8057.

[19] Sze SM, Ng KK. Physics of semiconductor devices. John wiley \& sons; 2006.

[20] Wang F, Shan J, Knoesel E, Bonn M, Heinz TF. Electronic charge transport in sapphire studied by opticalpump/THz-probe spectroscopy. In: Ultrafast Phenomena in Semiconductors and Nanostructure Materials VIII 2004 (Vol. 5352). International Society for Optics and Photonics. 2004. p. 216-221.

[21] Look DC. Electrical and optical properties of p-type ZnO. Semiconductor Science and Technology. 2005;20(4):S55. 
[22] Whitaker J. Electrical properties of n-type aluminium arsenide. Solid-State Electronics. 1965;8(8):649-652.

[23] Eaton SM, Hadden JP, Bharadwaj V, Forneris J, Picollo F, Bosia F, Sotillo B, Giakoumaki AN, Jedrkiewicz O, Chiappini A, Ferrari M. Quantum micro-nano devices fabricated in diamond by femtosecond laser and ion irradiation. Advanced Quantum Technologies. 2019:1900006.

(C) 2019 by the author(s). This work is licensed under a Creative Commons Attribution 4.0 International License (http://creativecommons.org/licenses/by/4.0/). Authors retain copyright of their work, with first publication rights granted to Tech Reviews Ltd. 